
\title{
28 Research Suare \\ Large-scale in vitro cultivation of Plasmodium falciparum under atmospheric air
}

\section{Crispim}

Department of Parasitology, Institute of Biomedical Science, University of São Paulo

\section{Ignasi Bofill Verdaguer}

Department of Parasitology, Institute of Biomedical Science, University of São Paulo

\section{Sofia F Silva}

University of São Paulo Institute of Biomedical Sciences: Universidade de Sao Paulo Instituto de Ciencias Biomedicas

\section{Alejandro Miguel Katzin ( $\square$ amkatzin@icb.usp.br)}

Institute of Biomedical Science, Univerity of São Paulo https://orcid.org/0000-0002-4782-2162

\section{Methodology}

Keywords: Plasmodium falciparum, culture, atmospheric air, drug screening, parasite viability

Posted Date: September 2nd, 2020

DOI: https://doi.org/10.21203/rs.3.rs-68992/v1

License: (c) (1) This work is licensed under a Creative Commons Attribution 4.0 International License. Read Full License 


\section{Abstract}

Background: The most important tool for the study of the malaria parasite is probably the in vitro culture that was established in 1976 by Trager and Jensen. However, the methodology is technically complex, expensive, and unsuitable for use in laboratories and regions with limited resources. Human factors such as hormones and the immune system are frequently ignored, and the similarity between the in vitro culture conditions and physiologic-like environment has been questioned. One of the most controversial factors about Plasmodium culture is the gaseous composition employed in vitro, which consists of a mixture poor in $\mathrm{O}_{2}(1 \%-5 \%)$ and rich in $\mathrm{CO}_{2}$ (approximately $5 \%$ ).

Results: The requirement of employing commercial gaseous mixtures and their suitability for use in reusable glass bottles under agitation for culturing were assessed. The methodology presented here is suitable for small- and large-scale culturing in cell culture flasks or hermetic and sterile glass bottles in agitation and provides an easier and cheaper Plasmodium culture alternative, which dispenses several sophisticated technical requirements of the classical methodology. The parasite viability, free oxygen, and drug-screening assays under these conditions were compared with those in the classic conditions. The cultivation under atmospheric air did not substantially affect the free oxygen levels and parasitic proliferation for periods longer than two years. However, it changed atovaquone, artesunate, and chloroquine efficacy in a different ways across the different isolates. Oxygraphic experiments demonstrated that the free oxygen concentration contained in the media was slightly superior compared to both the human blood and the media employed the classical methodology.

Conclusions: The results indicate that the described culture conditions are suitable for parasite maintenance and can be used to obtain high yields of parasite. Although parasites proliferate under various culture conditions, it is clear that it is necessary to evaluate the different gaseous conditions to validate an antimalarial candidate. With this, it will be possible to detect an oxygen level that encompasses the physiological oxygen levels of human blood. Since oxygen decreases through media layers, future studies should further explore the use of oxygen-rich mixtures as a strategy to expose static cultures to more physiologic-like conditions.

\section{Background}

Malaria is a prevalent parasitic disease in Africa, Southeast Asia and South America, affecting 216 million people in 2018 and has caused approximately 445000 deaths mostly due Plasmodium falciparum infections in Africa [1]. Unfortunately, malaria is endemic in several undeveloped regions where there are few economic resources for treatment and epidemiologic control [2,3]. Moreover, malaria control presents other challenges including the major issue of drug-resistance [1, 4]. That's why the discovery of new antimalarial compounds, a better understand of the parasite's metabolism, and assessment of the drug-resistance spreading is required $[1,4]$. In this vein, interdisciplinary efforts are need to fight against malaria at a global scale. 
Central to the study of infectious diseases is the in vitro culturing of each etiologic agent. Briefly, it means to cultivate pathogens ex vivo as similarly as possible to their natural environments to increase the biological relevancy of the results. The Plasmodium culture was established in 1976 by Trager and Jensen, allowing the creating of an artificial, continuous intraerythrocytic cycle through the infection of red blood cells by the cultured parasites [5]. This method is one of the most widely-used tools for the study of parasites [5] such as epidemiologic studies focused on isolating field isolates [6] for in vitro drug screening the study of the parasitic metabolism and for studies requiring large amounts of parasites.

The Trager and Jensen culture methodology was based on the use of a candle jar and/or cell flasks containing human red blood cells suspended in culture medium and a specific gaseous mixture rich in $\mathrm{CO}_{2}$ and poor in $\mathrm{O}_{2}$ [5]. These gaseous conditions were obtained by direct gas injection or by candle combustion [5]. The culture medium used was composed of RPMI medium 1640 (Thermo Fisher Scientific, Waltham, Massachusetts, U.S.) complemented with $0.1 \mathrm{~g} / \mathrm{L} \mathrm{p}, 2 \mathrm{~g} / \mathrm{L}$ glucose, $0.04 \mathrm{~g} / \mathrm{L}$ gentamicin, and $5 \mathrm{~g} / \mathrm{L}$ of Albumax I (Thermo Fisher Scientific, Waltham, Massachusetts, U.S.). Some authors have attempted to optimize the primogenital culture methodology: the most important modifications were the substitution of human plasma for commercial Lipid-Rich Bovine serum albumin [7] and the parasitic stage synchronization by the D-sorbitol techniques [8, 9]. Preechapornkul et al in 2010, used bioreactors under constant agitation and controlled atmosphere to optimize the Plasmodium culture [10]. Moreover, Radfar et al combined new techniques and standardized very intricate protocols to scale-up the culturing for large scale parasite recovery [11]. Unfortunately, the last two studies describe culturing methodology that is not suitable for research groups in areas lacking technical and economic resources, a frequent situation in areas endemic for malaria.

An important requirement for in vitro culture is the ability to mimic physiologic conditions, allowing extrapolations of the biological reality. Human factors such as temperature changes and immune responses are relevant differences between Plasmodium cultures and the human infection [12]. However, the most controversial factor reported about Plasmodium cultures are the in vitro gaseous composition employed [12].

Different gaseous mixtures were employed across the studies and marginal effects on the parasitic viability [13] and drug susceptibility [14], were identified, however the relevance of gas mixtures remains unclear.

Here, we present a new and simple methodology, suitable for the culture of Plasmodium falciparum at a small- or large-scale. Though the techniques used we have employed have previously been established, our protocol drastically reduces the expenses, resource-allocation, and use of specialized equipment, enabling easier Plasmodium culturing. By administering atmospheric air (ATM) we show similar outcomes regarding parasite viability and some drug-screening, compared with the classic culturing methodology. Our protocol simplifies the in vitro culturing of Plasmodium and provides a new, standardized method for use by researcher in this field. In subsequent sections, we discuss the different culturing methodologies in literature as well as researcher opinions regarding the applicability and 
biological relevance of the various in vitro culturing techniques with respect to the natural environment of the parasite.

\section{Results}

\section{Influence of gas mixtures and cultivation method on parasite growth in vitro}

Three $P$. falciparum isolates were cultivated in cell flasks under the classical mixture of gas described by Trager and Jensen or ATM. The parasitemia of the cultures were recorded for at least 5 days and as can be seen in Fig. 1a-d. There were no differences in $P$. falciparum proliferation neither the proportion on intraerythrocytic stages. This result was confirmed in the two methodologies used to measure the parasitemia. Inferior ratios of parasitic growth were only observed while employing an initial parasitemia $<0.5 \%$. It is important to note that there were other cultures in our laboratory that have been kept for over two years in the absence of gas mixture or candle jar. In addition, other cultures were also kept in ATM but stirring in $2,1,0.5,0.25$ or $0.1 \mathrm{~L}$ glass bottles as described in materials and methods. In all cases no differences on parasitic proliferation neither the proportion on intraerythrocytic stages were observed as can be seen in Fig. 1 (a to $c$ shows results obtained by employing $0.1 \mathrm{~L}$ bottles as an example) and in the representative images of Fig. 2.

Finally, oxymetric measurements of uninfected erythrocytes maintained in the same conditions of parasite culture showed that free oxygen levels in ATM $(\sim 180 \mu \mathrm{M})$ are superior in comparison to classic conditions $(\sim 147 \mu \mathrm{M})$ (Fig. 1e).

\section{Influence of $\mathrm{O}_{2}$ tension on antimalarial drugs activity in vitro}

In Fig. 3, we show that increasing $\mathrm{O}_{2}$ levels chloroquine (CQ) become more effective in the three isolates used. Interestingly, this phenomenon was much more evident for isolates NF54 and 3D7 in which CQ IC 50 value decreases $x 18$ and $x 13$ fold respectively. However, under identical conditions $C Q I_{50}$ value only decreases $\times 3$ fold in the K1 isolate. Moreover, NF54 isolate showed sensibility to CQ only when employing ATM ( $\mathrm{x} 10$ fold more sensitive than $\mathrm{K} 1$, a CQ-resistant isolate) but under classical conditions it is just $\mathrm{x} 1.7$ fold more sensible than $\mathrm{K} 1$. Under classic conditions all isolates have $\mathrm{AV}^{\mathrm{I}} \mathrm{C}_{50}$ value between $0.11-$ $0.37 \mathrm{nM}$ and under ATM the $\mathrm{IC}_{50}$ values increase but in a much different proportion: $\times 3$ fold for 3D7 isolate, $\mathrm{x} 33$ fold for $\mathrm{K} 1$ and $\mathrm{x} 80$ fold for NF54. Finally, under classic conditions, ART antiplasmodial effect does not show differences between the isolates but the introduction of ATM increases the effectiveness by $\mathrm{x} 10$ in the $\mathrm{K} 1$ isolate, decreases effectiveness by $\mathrm{x} 10$ in 3D7 and was statistically equal to classic conditions for NF54. It must be noted that under classic conditions the most ART-sensible isolate was NF54 followed by K1 and 3D7 while under ATM the most ART-sensible was 3D7 followed by NF54 and consecutively the $\mathrm{K} 1$ isolate.

The sigmoidal dose-response effects of all antimalarial under all conditions can be shown in Figs. 4 and 5 and the $\mathrm{IC}_{50}$ values were summarized in Table 1. 
Table 1

Summary of all $\mathrm{IC}_{50}$ data exposed to the classic low oxygen mixture and atmospheric air.

\begin{tabular}{|llll|}
\hline $\mathbf{5 \%} \mathbf{O}_{2}$ & & & \\
\hline Drug & $3 \mathrm{D} 7$ & $\mathrm{~K} 1$ & $\mathrm{NF54}$ \\
\hline AV & $0.37 \pm 0.18$ & $0.31 \pm 0.30$ & $0.11 \pm 0.05$ \\
CQ & $9.22 \pm 2.32$ & $231.63 \pm 43.65$ & $132.73 \pm 16.69$ \\
ART & $9.40 \pm 2.66$ & $4.59 \pm 0.53$ & $3.65 \pm 1.22$ \\
\hline ATM & & & \\
\hline Drug & $3 D 7$ & $\mathrm{~K} 1$ & NF54 \\
AV & $1.08 \pm 0.05$ & $9.95 \pm 0.16$ & $8.93 \pm 2.00$ \\
\hline CQ & $0.69 \pm 0.07$ & $77.20 \pm 5.64$ & $7.33 \pm 0.80$ \\
\hline ART & $0.98 \pm 0.29$ & $44.67 \pm 2.08$ & $2.22 \pm 0.52$ \\
\hline
\end{tabular}

\section{Discusion}

The Trager and Jensen gaseous mixtures containing $5 \% \mathrm{O}_{2}$ are defined as being normoxic, with respect to the natural gaseous composition of tissues [5] and they are widely used in malaria studies. Importantly, the $5 \% \mathrm{O}_{2}$ refer to the gaseous conditions of the culture vessels and/or incubators, however, the pericellular environment of the red blood cells may not present to the same gaseous condition. Before oxygen reach the cells, it must be exchanged at the surface of the media and cross the liquid layer in a process which is dependent on several factors such as $\mathrm{pH}$, cell density, pressure and medium volume and composition [15]. Notably, oxygen travels approximately 30 millimeters to reach cells in culture, whereas it travels approximately $10-30 \mu \mathrm{m}$ in animal tissues [16]. Once the cells are consuming oxygen, its diffusion must exceed consumption to avoid hypoxia.

Branco and Francisco previously discussed ${ }^{[12]}$ that is difficult to reproduce physiologic-like conditions in vitro, because of the large differences between the $\mathrm{O}_{2}$ saturations across human tissues, where some tissues can experience $10-13 \% \mathrm{O}_{2}$ saturation. However, considering that arterial oxygen concentration is approximately $130 \mu \mathrm{M}$ [15], our results indicate that the parasite statically cultured in vitro is exposed to slightly hyperoxia by employing both ATM and classic conditions. It should be noted that here it was assessed the free oxygen content in homogenized uninfected erythrocytes; the oxygen which reaches erythrocytes at the bottom of the flask probably is lower, quickly consumed by parasites or bounded to hemoglobin. Exemplifying, in another cells cultures in vitro, a fourfold decrease in $\mathrm{O}_{2}$ concentration respect the gas-liquid interphase has been previously reported [19]. Similarly to Branco and Francisco did in 2018 [12], here we purpose that to use oxygen rich mixtures may create a pericellular $\mathrm{O}_{2}$ 
concentration more similar to the natural environment of cells. Whatever the real $\mathrm{O}_{2}$ levels available for Plasmodium cultured in vitro is, it should be noted that different gaseous mixtures have already proven to maintain parasite viability. These gaseous mixtures include ATM (approximately $78.09 \% \mathrm{~N}_{2}, 20.95 \% \mathrm{O}_{2}$, $0.93 \mathrm{CO}_{2}$ and $0.04 \%$ other residual gases), high oxygen mixtures [14] or even microaerophilic environments [18]. Therefore, if it's unknown the parasitic natural gaseous conditions in human and if it's actually viable to maintain the parasites by employing ATM, why are we not maintaining the $P$. falciparum cultures in ATM? All results here presented, show that the cultivation under ATM did not affect the parasitic proliferation and parasitic stages proportion during the studied periods. Furthermore, cultures of $P$. falciparum 3D7 isolate have been maintained under ATM in our group for more than two years, which demonstrates the technique suitability for extended periods.

Once exposed the success by cultivating parasites in shaking and under ATM, some questions must be discussed about the cons to not follow the classic methodology. It could be said that using ATM or oxygen-rich environments occurs decreases on parasitic viability $[13,14]$. However, a decrease in parasitic viability under ATM has just been observed at low parasitemia levels $(<1 \%)$ [13] which could create difficulties in parasitic growth tests by $\left[{ }^{3} \mathrm{H}\right]$-hypoxanthine incorporation or DNA staining $[20,21]$.

We need to also consider the effects of antimalarial compounds on parasitic growth when modifying gaseous conditions in culture. Our findings demonstrated that those changes in the oxygen tension strongly modulate the antimalarials effect in a very different proportion across the isolates. Considering the question of whether pericellular oxygen in culture is similar to in vivo conditions in humans, our results suggest that it is not. This supports the idea that many reported drug-effects against parasites might not be extrapolable to their behavior and/or response in their natural environments, negatively impacting numerous studies in the literature. For example, many studies which showed promissory therapeutic indices in vitro might not be accurate. Similarly, another confounding variable might be the parasitic models (field isolates vs. obtained by mutagenesis) used in these studies for identification of drug resistant/sensible. The purpose of this study was to generate a culturing methodology that had low technical difficult and improve the environmental similarity to the parasitic environment.

From a biochemical perspective, differences in the antimalarial effects under different gaseous conditions elucidate mechanisms of action. CQ induces oxidative stress to the parasites [22], and our results suggest that oxygen-rich environments potentiate reactive oxygen species, consequently damaging the cells. Alternatively, since AV acts as a mitochondrial uncoupler [23] its antiplasmodial efficacy would improve as the mitochondrial activity diminishes due to the lack of oxygen. Both hypotheses were extracted by alternating the gaseous conditions. These kinds of studies can be interesting tools to better understand the parasitic metabolism and mechanisms of action of different drugs. Our group has previously employed non-classical gaseous mixtures $\left(0 \%\right.$ or $\left.20 \% \mathrm{O}_{2}\right)$ for these purposes $[17,18,24]$. In both cases, we observed parasite viability for at least 2-3 weeks under different gaseous mixtures and the biosynthesis of some substances directly involved in the respiration or oxidative stress defense such as ubiquinone and tocopherols $[17,18,25]$. The changes in the 
biosynthesis of these metabolites were attributed to the gaseous alterations. This permitted us to better understand both the biochemical mechanism in the parasite as well as the metabolic adaptations to different environments associated with different vertebrate host localizations.

Besides the effects of gaseous composition, other aspects of the culture methodology should also be reviewed. In our opinion, one important aspect that is often neglected is the in vitro chemical environments of parasites are cultured in. In this sense, several human-blood metabolites have demonstrated the ability to interfere on the antimalarials' activity. Despite this, these metabolites are not added to media or supplemented at physiologic concentrations. For example, vitamin $\mathrm{E}$ is not added to RPMI but it is established that it protects the parasite from the oxidative stress produced by CQ [26]. Another example is related the folate metabolism. Studies (both in vivo and in vitro) have demonstrated how p-aminobenzoic acid (pABA) and 4-hidroxybenzoate (4-HB) can rescue the antiplasmodial effect of some antifolate antibiotics $[27,28]$. However, $4-\mathrm{HB}$ is not added to commercial RPMI-1640 and pABA is added at approximately $7 \mu \mathrm{M}$, which is a much lower concentration than seen in blood (approximately $32 \mu \mathrm{M})$.

\section{Conclusions}

In this work, we showed a continuous culture of $P$. falciparum in a shaker with reusable-glass bottles, instead of using a sophisticated apparatus. With this methodology, it is possible to easily cultivate large volumes of cultures, with high parasitic yields $\left(1 \times 10^{10}\right.$ parasites in a few $1 \mathrm{~L}$ bottles). Our protocol decreases the technical difficulty of culturing and increases the yield of biological material for research groups lacking space and/or economic sources. Additionally, shaking cultures is another interesting option for increasing the oxygen diffusion to generate environments that more closely mimic the physiologic-like conditions.

\section{Methods}

\section{Reagents}

Albumax I and RPMI-1640 were purchased from Fisher Scientific ${ }^{\circledR}$ (Leicestershire, UK). A gaseous mixture of $5 \% \mathrm{CO}_{2}, 5 \% \mathrm{O}_{2}$, and $90 \% \mathrm{~N}_{2}$ was purchased from Air Products Brasil LTDA (São Paulo, SP, Brazil). Other reagents including saponin, hypoxanthine, gentamycin sulfate, D-sorbitol, glucose, 4-(2hydroxyethyl)-1-piperazine ethanesulfonic acid (HEPES), atovaquone (AV), chloroquine (CQ), and organic solvents were purchased from Sigma-Aldrich (St. Louis, Missouri, EUA). The artesunate (ART) and SYBR Green $I \circledast$ nucleic acid gel stain were purchased from the Guiling Pharmaceutical Factory (Guiling City, 21 Guangxi, China) and Life technologies ${ }^{\circledR}$ (Eugene, OR, USA), respectively.

Plasmodium falciparum intraerythrocytic stages culture

Basic requirements for Plasmodium culture 
Throughout this work, we used three different $P$. falciparum isolates: NF54 (drug sensible clone obtained from cases of malaria imported into the Netherlands) [29], K1 (isolated in Thailand and reported to be chloroquine-resistant) [30] and 3D7 (derived from NF54 by limiting dilution) [31]. Parasites were cultured at $37^{\circ} \mathrm{C}$ in RPMI- 1640 medium solved in ultra-pure in Milli-Q water (Millipore, Burlington, Massachusetts, EUA) and supplemented with $25 \mathrm{mM}$ HEPES, $0.5 \%$ Albumax $I^{\circledR}, 370 \mu \mathrm{M}$ hypoxanthine, $2 \mathrm{~g} / \mathrm{L}$ glucose and $25 \mathrm{mg} / \mathrm{L}$ gentamicin sulfate [11]. The $\mathrm{pH}$ of the media was adjusted to 7.4 with a $10 \% \mathrm{NaHCO}_{3}$ sterile solution and then it was filtered in Millipore Express ${ }^{\text {TM }}$ PLUS $0.22 \mu \mathrm{m}$ sterile filters. The reference gaseous mixture was composed of $5 \% \mathrm{CO}_{2}, 5 \% \mathrm{O}_{2}$, and $90 \% \mathrm{~N}_{2}$ [11], however, ATM was also employed when indicated. To avoid culture contamination, mycoplasma testing was done regularly and optic microscopy was performed daily [32]. Leukodepleted blood was a gift from Sírio Libanês Hospital (NESTA, São Paulo, Brazil) blood bank.

\section{Trager \& Jensen-based culture}

The P. falciparum intraerythrocytic stages at $2 \%$ hematocrit were cultured in vitro according to Trager \& Jensen [5] with modifications [11] in RPMI-1640 complete culture medium in 25 or $75 \mathrm{~cm}^{3}$ sterile cell culture flasks purchased from Corning ${ }^{\circledR}$ (Corning, NY, EUA). Culture changes were performed as described by Radfar et. at. [11], the reference gaseous mixture or ATM were employed when indicated. Parasitic proliferation was studied by microscopy of Giemsa-stained smears and statistically analyzed to compare the parasitic growth/stages under different culture methodologies or conditions by a one-way ANOVA/Tukey multiple comparison test.

\section{Culture in glass bottles}

Parasites were cultured in $2,1,0.5,0.25$, or $0.1 \mathrm{~L}$ Schott hermetic and sterile glass bottles (Schott Glas, Mainz, Germany) containing and $20 \%$ of its volume of $2 \%$ hematocrit culture as described. Parasites were cultivated at $37^{\circ} \mathrm{C}$ and under orbital agitation (130 RPM) in a Thermo Scienific MaxQ 6000 shaker (model 4353). In this methodology, it was employed ATM. Culture medium composition and changes were performed as described in last item.

\section{Parasitic synchronization at ring stage}

Culture synchronization at ring stage (6-22 $\mathrm{h}$ after the invasion) was performed as described by $\mathrm{C}$. Lambros \& Vanderberg [8]. Briefly, cultures were centrifuged at $200 \times \mathrm{g}$ for $5 \mathrm{~min}$ and synchronization was

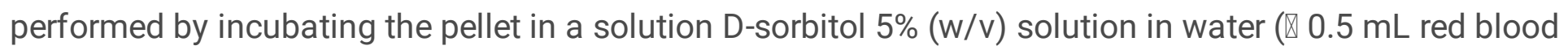
cells/2.5 mL D-sorbitol solution).

\section{Drug assays}

Drug experiments that required the measurement of parasite proliferation were performed in 96-well plates contained in hermetic boxes for plate incubation. The boxes were filled with the indicated gaseous mixture or maintained in ATM. All assays started at ring stage ( $2 \%$ parasitemia, $2 \%$ hematocrit) and parasite growth was monitored after $48 \mathrm{~h}$ by both Giemsa-stained smears and DNA staining [20]. The compound concentration which decreases at $50 \%$ of parasitic growth $\left(\mathrm{IC}_{50}\right)$ at $48 \mathrm{~h}$ was calculated 
following the Desjardins et al. methodology [33]. Briefly, 1:1 (vol:vol) serial dilution was performed to obtain a decreasing drug concentration in the 96-well plate. An initial concentration of $400 \mathrm{nM}$ for AV and $1000 \mathrm{nM}$ for CQ or ART was selected. Comparison with untreated controls and a solvent control was always performed. Inhibition of parasitic growth was analyzed in relation to the logarithm of the concentration of the compound using a nonlinear regression (dose-response slope/variable sigmoid equation). The parasite $\mathrm{IC}_{50}$ analysis is performed using GraphPad Prism® software (GraphPad Software ${ }^{\circledR}$, Inc., California, USA). It is also calculated the R-squared value $\left(R^{2}\right)$ for the dose-response and oonly those assays showing $\mathrm{R}^{2}>0.95$ were accepted. All experiments were repeated at least three times with three technical replicates.

\section{Parasitic growth monitoring}

Growth was monitored after $48 \mathrm{~h}$ by Giemsa stained smears by optical microscopy or DNA staining for all experiments. For DNA staining, we followed procedures described by Smilkstein et al. [20]. An amount of $100 \mu \mathrm{L}$ of parasite culture was incubated in a 96-well cell plate in darkness and at room temperature after the addition of $100 \mu \mathrm{L}$ of Syber Green I® 2/10.000 (v/v) in lysis buffer [20 mM Tris, pH 7.5; 5 mM EDTA; $0.008 \%$ saponin $(\mathrm{w} / \mathrm{v}) ; 0.08 \%$ Triton $\mathrm{X}-100(\mathrm{v} / \mathrm{v})]$. We used uninfected erythrocytes as blanks at the same hematocrit and subtracted its fluorescence measurements from the readings of the samples. Fluorescence was measured using POLARstar Omega fluorometer ${ }^{\circledR}$ (BMG Labtech ${ }^{\circledR}$, Ortenberg, Germany) at the excitation and emission wavelengths of 485 and $520 \mathrm{~nm}$, respectively.

\section{Oxygraphy}

Red blood cells were suspended at $2 \%$ hematocrit in complete RPMI medium and maintained under classic gaseous mixture or ATM for $24 \mathrm{~h}$ at $37^{\circ} \mathrm{C}$. Then, the sample was homogenized and the oxygen content in $2 \mathrm{~mL}$ was assessed at $37^{\circ} \mathrm{C}$ in a high-resolution oxygraph (Oxygraph-2 k Oroborus Instruments, Innsbruck, Austria).

\section{Declarations}

\section{ETHICS APPROVAL AND CONSENT TO PARTICIPATE}

Not applicable.

\section{CONSENT FOR PUBLICATION}

Not applicable.

\section{AVAILABILITY OF DATA AND MATERIALS}

Please contact the author for data requests.

\section{COMPETING INTERESTS}

The authors declare that they have no competing interests. 


\section{FUNDING}

Crispim M. and Verdaguer I.B are fellows at the Fundação de Amparo à Pesquisa do Estado de São Paulo, Brasil (FAPESP). This research was supported by grants from the FAPESP and Conselho Nacional de Desenvolvimento Científico e Tecnológico Brasil (CNPq).

\section{AUTHORS' CONTRIBUTIONS}

$\mathrm{MC}$ and IBV contributed equally in conceptualization, formal analysis, investigation, methodology and writing. SFS contribute in investigation and methodology, AMK contributed in conceptualization, data curation, formal analysis, funding acquisition, investigation, project administration, resources, supervision and writing - review \& editing.

\section{ACKNOWLEDGEMENTS}

The authors would like to thank Prof. Dr. William Tadeu Lara Festuccia and Mr. Érique de Castro from the "Laboratório de Fisiologia Molecular e Metabolismo" in ICB/USP, for providing the platform and helping with oximetry tests and Hospital Sírio-Libanês for gifting the erythrocytes for our study.

\section{Abbreviations}

4-HB: 4-hydroxybenzoate

ART: Artesunate

ATM: Atmospheric air

AV: Atovaquone

CF: Culture flasks

CQ: Chloroquine

GB: Glass bottles

HEPES: 4-(2-hydroxyethyl)-1-piperazine ethanesulfonic acid

$\mathrm{IC}_{50}$ : half maximal inhibitory concentration

pABA: p-aminobenzoic acid

\section{References}

1. World Health Organization. The World malaria report 2019. WHO. 2019;1:232. 
2. Gallup JL, Sachs JD. The economic burden of malaria. Am J Trop Med Hyg. 2001. doi:10.4269/ajtmh.2001.64.85.

3. Foster S, Phillips M. Economics and its contribution to the fight against malaria. Ann Trop Med Parasitol. 1998;92:391-8.

4. WHO. Malaria drug resistance. Bull World Health Organ. 2000;78:407.

5. Trager W, Jensen JB. Human malaria parasites in continuous culture. Science. 1976;193:673-5.

6. Penh P, Thomas SM, Ndir O, Dieng T, Mboup S, Wypij D, et al. In vitro chloroquine susceptibility and PCR analysis of pfcrt and pfmdr1 polymorphisms in Plasmodium falciparum isolates from Senegal. Am J Trop Med Hyg. 2002;66:474-480.

7. Cranmer SL, Magowan C, Liang J, Coppel RL, Cooke BM. An alternative to serum for cultivation of Plasmodium falciparum in vitro. Trans R Soc Trop Med Hyg. 1997;91:363-5.

8. Lambros C, Vanderberg JP. Synchronization of Plasmodium falciparum Erythrocytic Stages in Culture. J Parasitol. 1979;65:418-42.

9. Srinivas SD, Puri SK. Time course of in vitro maturation of intra-erythrocytic malaria parasite: a comparison between Plasmodium falciparum and Plasmodium knowlesi. Mem Inst Oswaldo Cruz. 2002;97:901-3.

10. Preechapornkul P, Chotivanich K, Imwong M, Dondorp AM, Lee SJ, Day NPJ, et al. Optimizing the culture of Plasmodium falciparum in hollow fiber bioreactors. Southeast Asian J Trop Med Public Health. 2010;41:761-9.

11. Radfar A, Méndez D, Moneriz C, Linares M, Marín-García P, Puyet A, et al. Synchronous culture of Plasmodium falciparum at high parasitemia levels. Nat Protoc. 2009;4:1899-915.

12. Branco A, Francisco D, Hanscheid T. Is there a "normal" oxygen concentration for in vitro Plasmodium Cultures? Trends Parasitol. 2018;34:811-2.

13. Mirovsky P. Continuous culture of Plasmodium falciparum asexual stages in "normal" air atmosphere. Folia Parasitol (Praha). 1989/01/01. 1989;36:107-112.

14. Briolant S, Parola P, Fusaï T, Madamet-Torrentino M, Baret E, Mosnier J, et al. Influence of oxygen on asexual blood cycle and susceptibility of Plasmodium falciparum to chloroquine: Requirement of a standardized in vitro assay. Malar J. 2007;6:44.

15. Place TL, Domann FE, Case AJ. Limitations of oxygen delivery to cells in culture: An underappreciated problem in basic and translational research. Free Radic Biol Med. 2017;113:31122.

16. Finch CA, Lenfant C. Oxygen Transport in Man. N Engl J Med. 1972;286:407-15.

17. Sussmann RACC, Angeli CB, Peres VJ, Kimura EA, Katzin AM. Intraerythrocytic stages of Plasmodium falciparum biosynthesize vitamin E. FEBS Lett. 2011;585:3985-91.

18. Tonhosolo R, Gabriel HB, Matsumura MY, Cabral FJ, Yamamoto MM, D’Alexandri FL, et al. Intraerythrocytic stages of Plasmodium falciparum biosynthesize menaquinone. FEBS Lett. 2010;584:4761-8. 
19. Newby D, Marks L, Lyall F. Dissolved oxygen concentration in culture medium: Assumptions and pitfalls. Placenta. 2005;26:353-7.

20. Smilkstein M, Sriwilaijaroen N, Kelly JX, Wilairat P, Riscoe M. Simple and inexpensive fluorescencebased technique for high-throughput antimalarial drug screening. Antimicrob Agents Chemother. 2004;48:1803-6.

21. Chulay JD, Haynes JD, Diggs CL. Plasmodium falciparum: Assessment of in vitro growth by $\left[{ }^{3} H\right]$ hypoxanthine incorporation. Exp Parasitol. 1983;55:138-46.

22. Sarin K, Kumar A, Prakash A, Sharma A. Oxidative stress and antioxidant defence mechanism in Plasmodium vivax malaria before and after chloroquine treatment. Indian J Malariol. 1993;30:12733.

23. Radloff PD, Philipps J, Nkeyi M, Hutchinson D, Kremsner PG. Atovaquone and Proguanil for Plasmodium falciparum Malaria. Infect Dis Clin Pract. 1996;347:1511-4.

24. Tonhosolo R, D’Alexandri FL, de Rosso VV, Gazarini ML, Matsumura MY, Peres VJ, et al. Carotenoid Biosynthesis in Intraerythrocytic Stages of Plasmodium falciparum. J Biol Chem. 2009;284:997485.

25. Verdaguer IB, Zafra CA, Crispim M, Sussmann RAC, Kimura EA, Katzin AM. Prenylquinones in human parasitic protozoa: Biosynthesis, physiological functions, and potential as chemotherapeutic targets. Molecules. 2019;24:3721.

26. Sussmann RACC, Fotoran WL, Kimura EA, Katzin AM. Plasmodium falciparum uses vitamin e to avoid oxidative stress. Parasites Vectors. 2017;10:461.

27. Nzila A. Inhibitors of de novo folate enzymes in Plasmodium falciparum. Drug Discov Today. 2006;11:939-44.

28. Taylor AE. The effect of paraminobenzoic acid, parahydroxybenzoic acid and riboflavin on Plasmodium gallinaceum in chicks. Trans R Soc Trop Med Hyg. 1957;51:241-7.

29. Ponnudurai T, Leeuwenberg DEM, Meuwissen Th. JHE. Chloriquine sensitivity of isolates of Plasmodium falciparum adapted to in vitro culture. Trop Geogr Med. 1981;33:50-4.

30. Agarwal P, Anvikar AR, Pillai CR, Srivastava K. In vitro susceptibility of indian Plasmodium falciparum isolates to different antimalarial drugs \& antibiotics. Indian J Med Res. 2017;146:622-8.

31. Walliker D, Quakyi IA, Wellems TE, McCutchan TF, Szarfman A, London WT, et al. Genetic analysis of the human malaria parasite Plasmodium falciparum. Science. 1987;236:1661-6.

32. Rowe JA, Scragg IG, Kwiatkowski D, Ferguson DJP, Carucci DJ, Newbold Cl. Implications of mycoplasma contamination in Plasmodium falciparum cultures and methods for its detection and eradication. Mol Biochem Parasitol. 1998;92:177-80.

33. Desjardins RE, Canfield CJ, Haynes JD, Chulay JD. Quantitative assessment of antimalarial activity in vitro by a semiautomated microdilution technique. Antimicrob Agents Chemother. 1979;16:710-8.

\section{Figures}


a

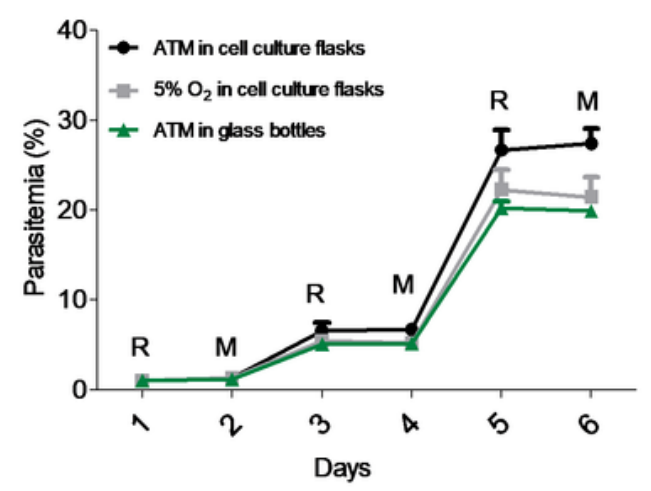

C

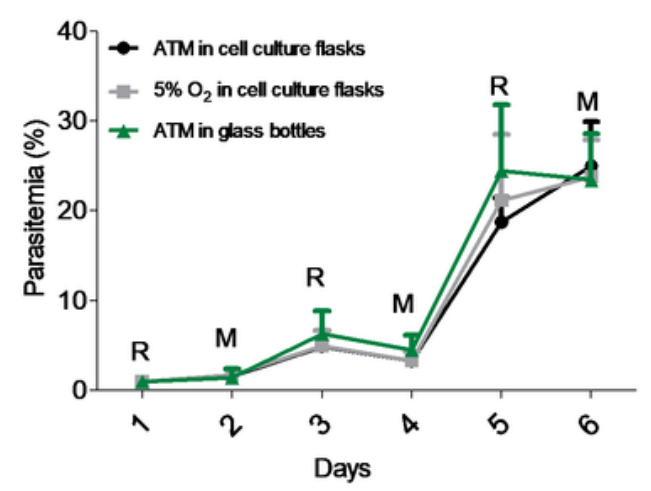

b

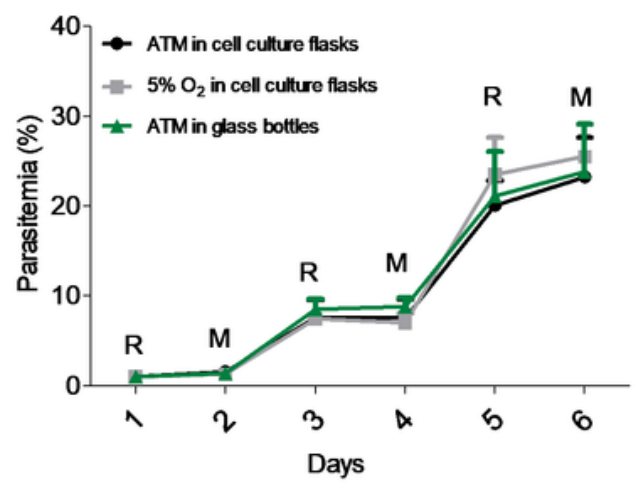

d

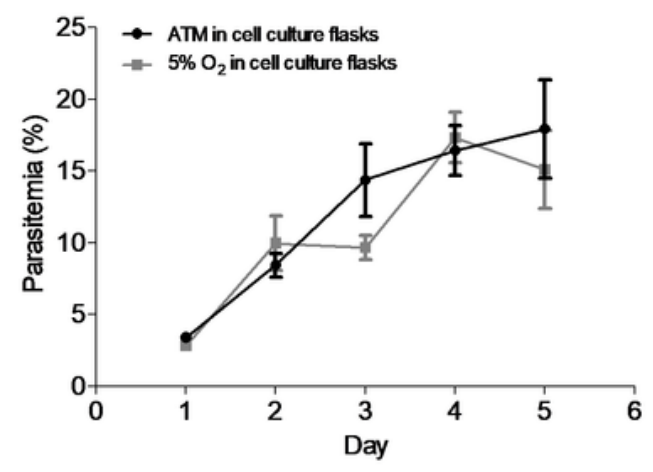

e

ATM

$5 \% \mathrm{O}_{2}$

Oxygen concentration $(\mu \mathrm{M})$

$180.54 \pm 4.14$

$147.36 \pm 0.25$

\section{Figure 1}

Parasite proliferation under distinct culture conditions. Cycle progression of parasites under different gas conditions employing cell culture flasks or $0.1 \mathrm{~L}$ sterile glass bottles. The P. falciparum isolates (a) 3D7, (b) K1, and (c) NF54 were pre-cultivated and synchronized. $2 \%$ hematocrit were infected reaching to $1 \%$ parasitemia (day 1). Parasitemia was measured daily using optical microscopy as previously described. In (d) the culture parasitemia of 3D7 isolate was measured daily using the methodology previously 
described by Smilkstein et al. [20]. ATM: atmospheric air, 5\% 02: low-oxygen gaseous mixture described by Trager and Jensen, R: ring stage, M: mature trophozoite/schizont forms. (d) Exposed free oxygen levels in uninfected cultures of erythrocytes maintained under different conditions.

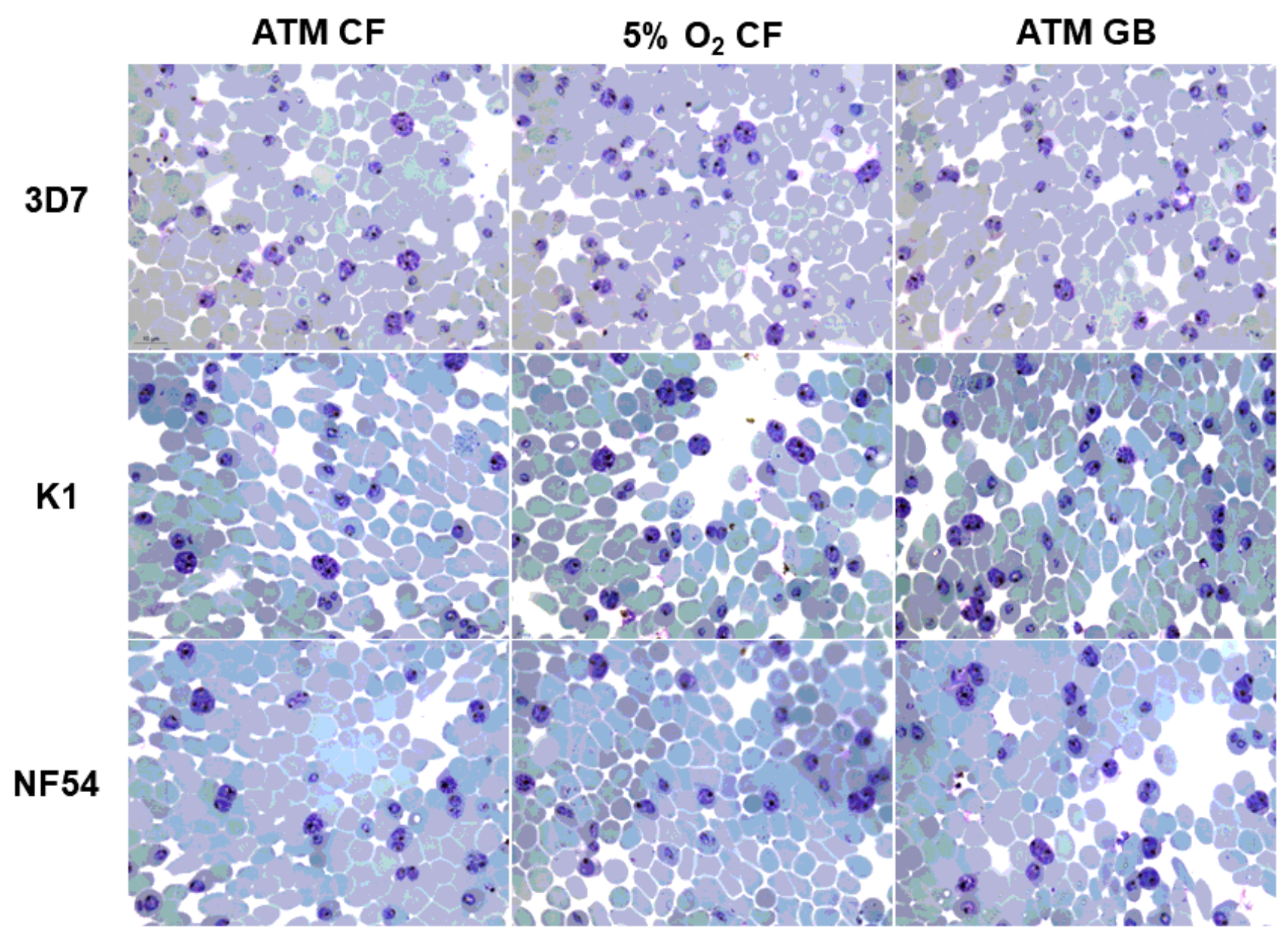

Figure 2

Representative pictures of P. falciparum schizonts of 3D7, K1 and NF54 at day 6 post-infection using optical microscopy. ATM CF: parasites cultivated using culture flasks under atmospheric air. $5 \% 02 \mathrm{CF}$ : parasites were cultivated using culture flasks under classical mixture of gas described by Trager and Jensen. ATM GB: parasites cultivated using glass bottles under atmospheric air. Cultures at $2 \%$ hematocrit were infected with $1 \%$ parasitemia (day 1 ). isolated 3D7, isolated K1, isolated NF54. 

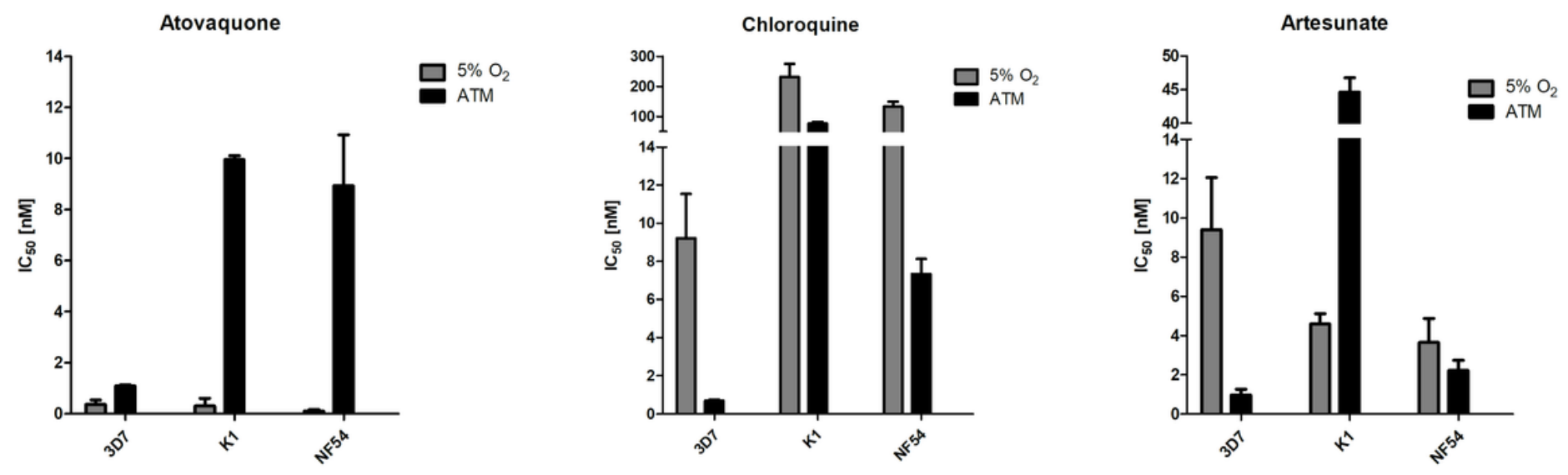

Figure 3

Effect of gaseous mixtures on antimalarial IC50. Effect of Atovaquone, Chloroquine, and Artesunate under classic low oxygen mixture versus ATM in 3D7, K1, and NF54 isolates.

\section{classic low oxygen mixture}
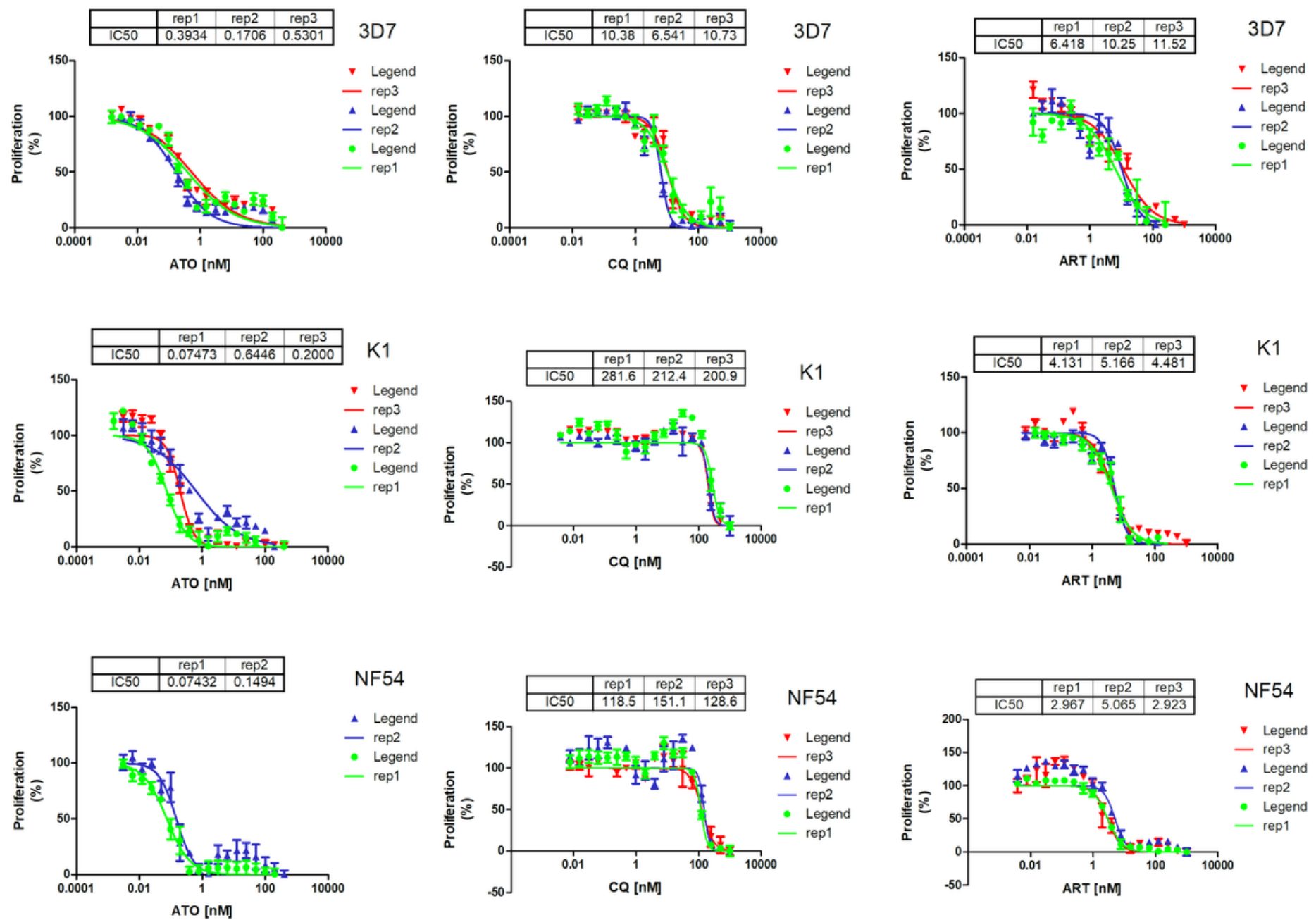
Figure 4

Sigmoidal dose-response effect of antimalarials under classic low oxygen mixture. Sigmoidal doseresponse effect of AV (Atovaquone), CQ (Chloroquine), and ART (Artesunate) in P. falciparum 3D7, K1, and NF54 isolates utilizing classic gaseous mixture established by Trager and Jensen. The parasites were cultivated for $48 \mathrm{~h}$ at $37^{\circ} \mathrm{C}$ in 96 well plates.

\section{Atmospheric gas}
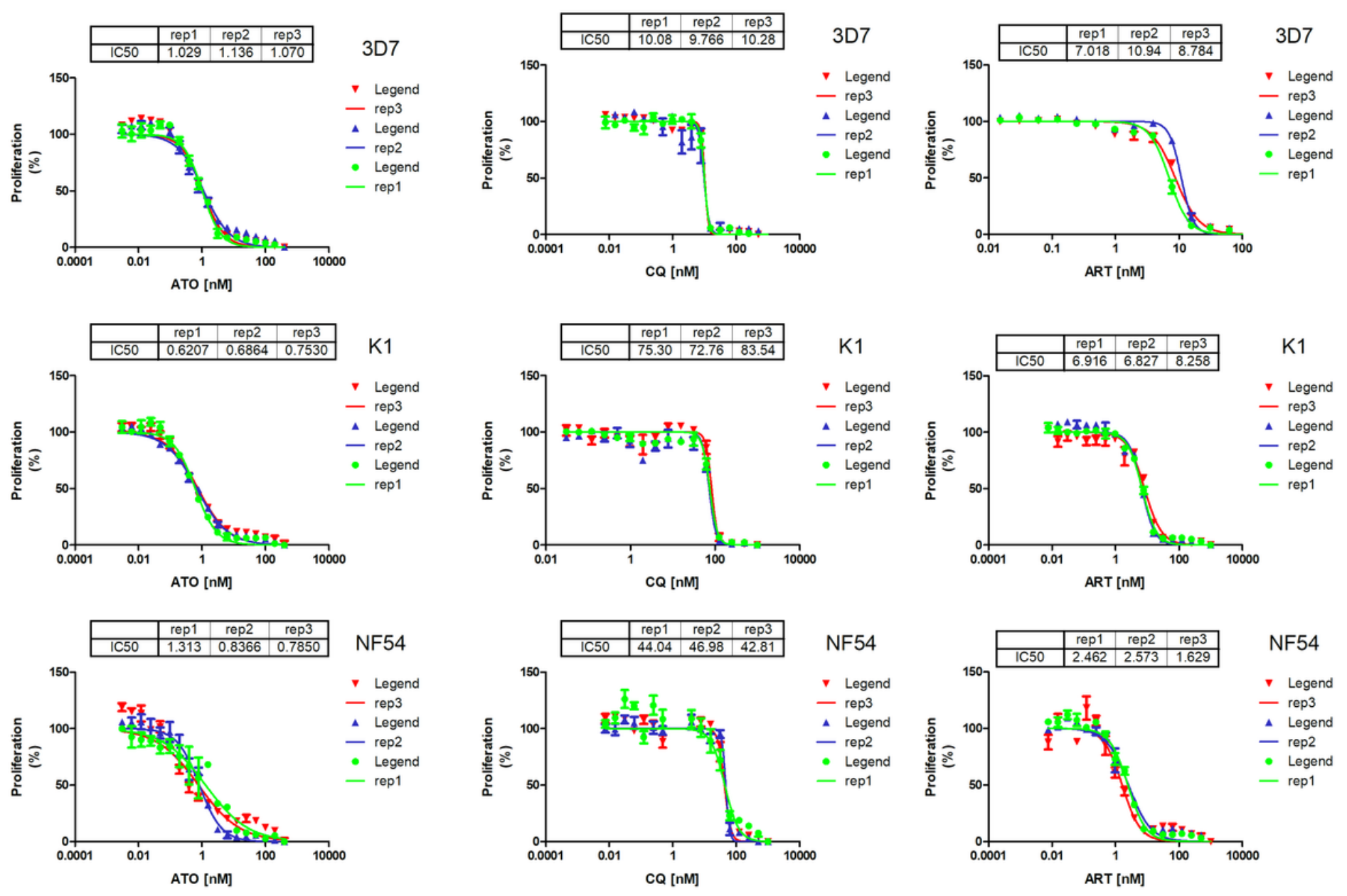

\section{Figure 5}

Sigmoidal dose-response effect of antimalarial under atmospheric air. Sigmoidal dose-response effect of AV (Atovaquone), CQ (Chloroquine), and ART (Artesunate) in P. falciparum 3D7, K1, and NF54 isolates without addition of gaseous mixture. The parasites were cultivated for $48 \mathrm{~h}$ at $37^{\circ} \mathrm{C}$ in 96 well plates. 